\title{
Clinical Presentation, Imaging, and Management of Segmental Arterial Mediolysis: A Rare Vascular Disorder
}

\author{
Ezhilmathi Alavandar ${ }^{1}$ Shobana Umapathy ${ }^{1}$ Santhosh Poyyamoli ${ }^{1}$ Venkatesh Kasi Arunachalam ${ }^{1}$ \\ Pankaj Mehta ${ }^{1}$ Mathew Cherian ${ }^{1}$ \\ ${ }^{1}$ Department of Radiology, Kovai Medical Center and Hospital, \\ Coimbatore, Tamil Nadu, India \\ Indian J Radiol Imaging 2021;31:983-989. \\ Address for correspondence Venkatesh Kasi Arunachalam, DMRD, \\ DNB, FRCR, Department of Radiology, KMCH, Avanashi Road, \\ Coimbatore, Tamil Nadu, 641014, India \\ (e-mail: radvenki79@gmail.com; drkasivenkatesh@yahoo.co.in).
}

\begin{abstract}
Keywords

- dissection

- embolization

- hemorrhage

- management

- segmental arterial mediolysis
\end{abstract}

\section{Introduction}

Segmental arterial mediolysis (SAM), which was first reported by Slavin and Gonzalez-Vitale in 1976, is a rare nonarteriosclerotic, noninflammatory vascular disease char- acterized by the disruption of the outer layer of the arterial media, which could lead to dissection, aneurysm, stenosis, and occlusion of the affected segment. ${ }^{1}$

The etiology of SAM is unknown, although vasospasm may play a role, as has been suggested by a few researchers. ${ }^{2-4}$ It is published online November 30, 2021
DOI https://doi.org/ 10.1055/s-0041-1736583. ISSN 0971-3026.

\footnotetext{
(C) 2021. Indian Radiological Association. All rights reserved. This is an open access article published by Thieme under the terms of the Creative Commons Attribution-NonDerivative-NonCommercial-License, permitting copying and reproduction so long as the original work is given appropriate credit. Contents may not be used for commercial purposes, or adapted, remixed, transformed or built upon. (https://creativecommons.org/ licenses/by-nc-nd/4.0/)

Thieme Medical and Scientific Publishers Pvt. Ltd., A-12, 2nd Floor, Sector 2, Noida-201301 UP, India
} 
important to distinguish SAM from other vascular pathologies like polyarteritis nodosa (PAN), Takayasu's arteritis, Henoch Schönlein purpura, Behçet's disease, fibromuscular dysplasia (FMD), mycotic aneurysms, and congenital vascular disorders.

SAM characteristically involves the splanchnic visceral arteries and the renal arteries, as well as the coronary and cerebral arteries to a lesser extent. The most common imaging findings include dissection, aneurysms, beading, occlusion and arterial wall thickening, which can result in ischemia/infarction of the involved organ or sometimes a life-threatening hemorrhage. A patient with SAM can be managed conservatively with antiplatelets/anticoagulants or by surgical/endovascular intervention if necessary. A high index of suspicion and exclusion of other differentials by clinical/laboratory data is necessary for a diagnosis of SAM to be made.

The primary aim of this study was to analyze the clinical presentations, imaging findings, and management of patients with SAM in different case scenarios within our institution.

\section{Materials and Methods}

Our study was approved by the Institutional Review Board and was conducted with the informed consent of the patients. A total of 13 patients, of which 12 were male, were included in the study, with their ages ranging from 29 to 75 years. We retrospectively analyzed all these 13 cases of SAM evaluated in our institution from July 2017 to March 2020. The images were collected from PACS along with other clinical information from the hospital's information system. Patients were selected based on clinical profile and imaging characteristics. All the patients we studied underwent contrast-enhanced computed tomography (CT) using a third-generation Siemens SOMATOM Force dualsource CT scanner. All the phases were acquired in singleenergy mode and scanning was performed after administering 70 to $80 \mathrm{~mL}(1-1.5 \mathrm{~mL} / \mathrm{kg})$ of nonionic IV contrast agent at an injection rate of 4 to $5 \mathrm{~mL} /$ second, with bolus tracking performed in the proximal abdominal aorta.

The dual-phase scanning included the late arterial and venous phases, which were acquired 15 to 20 seconds and 60 seconds after bolus tracking, respectively. Multiplanar reconstruction images in the coronal and sagittal planes, maximum intensity projection images, and volume-rendered images were generated and analyzed using the workstation's built-in syngo.via software.

\section{Results}

The most common clinical presentation of SAM in our study was abdominal pain, followed by flank pain with deranged renal function and intra-abdominal/retroperitoneal hemorrhage. Nine out of the 13 patients presented with abdominal pain, while two of them had flank pain with worsening renal function. The remaining two cases presented with intraabdominal/retroperitoneal hemorrhage.
The most common artery involved in our series was the celiac artery, which was involved in six cases. The involvement of the superior mesenteric and hepatic arteries was seen in four patients. The renal artery was involved in three patients, one of whom presented with retroperitoneal hematoma. The splenic artery was involved in two cases, while the left gastric artery and a jejunal branch of the SMA were both involved in one case.

The most common arterial imaging finding, which was seen in eight of the patients, was dissection with aneurysm/thrombosis/intramural hematoma. Circumferential wall thickening with luminal narrowing was seen in three patients. A beaded appearance of the vessel with alternate segments of narrowing and dilatation in the jejunal branch of the SMA was noted in one case. A focal fusiform/saccular aneurysm was noted in three patients in the celiac, SMA, and renal arteries. The imaging findings for selected patients are provided in - Figs. 1 to 4 .

We analyzed the clinical profile, history, and examination findings as well as the laboratory parameters of our patients, which included measuring the erythrocyte sedimentation rate, C-reactive protein, and the autoantibody panel, and excluded congenital structural vascular disorders, connective tissue disorders, and vasculitis.

Nine of our 13 patients were managed conservatively with antiplatelets/anticoagulants. Endovascular intervention was performed in four patients, two patients underwent stentgrafting of a celiac axis dissecting aneurysm, and one underwent stent-grafting of the renal artery. Embolization of one of the jejunal branches of the SMA was performed in a patient with mesenteric hematoma. All of the cases are summarized in - Table 1.

We conducted follow-up imaging on nine of the patients from a period of 2 months until 30 months after the initial treatment. The stabilization or regression of the imaging findings was documented in the follow-up scans and none of the patients exhibited any worsening in their condition.

\section{Discussion}

As mentioned earlier, SAM is a rare, noninflammatory vasculopathy that predominantly affects medium-sized vessels. The main pathology in SAM is secondary vacuolization and lysis in the outer layer of the arterial media, which causes the formation of arterial gaps and the loss of the external elastic lamina. This can then lead to dissecting hematomas and aneurysms at the site of the arterial gaps, which can present as intra-abdominal/retroperitoneal hemorrhage. ${ }^{1} \mathrm{~A}$ unique feature of the dissection caused by SAM is that it involves the outer layer of the media and adventitia rather than disrupting the intima. ${ }^{2-4}$ This acute phase is followed by a late reparative phase in which there is a deposition of granulation tissue that eventually leads to fibrosis and vessel remodelling. $^{2}$

The extant literature states that SAM usually affects middle-aged to elderly people with no gender predilection. ${ }^{3-6}$ However, the patients in our study were predominantly male. The clinical manifestations of SAM can be 


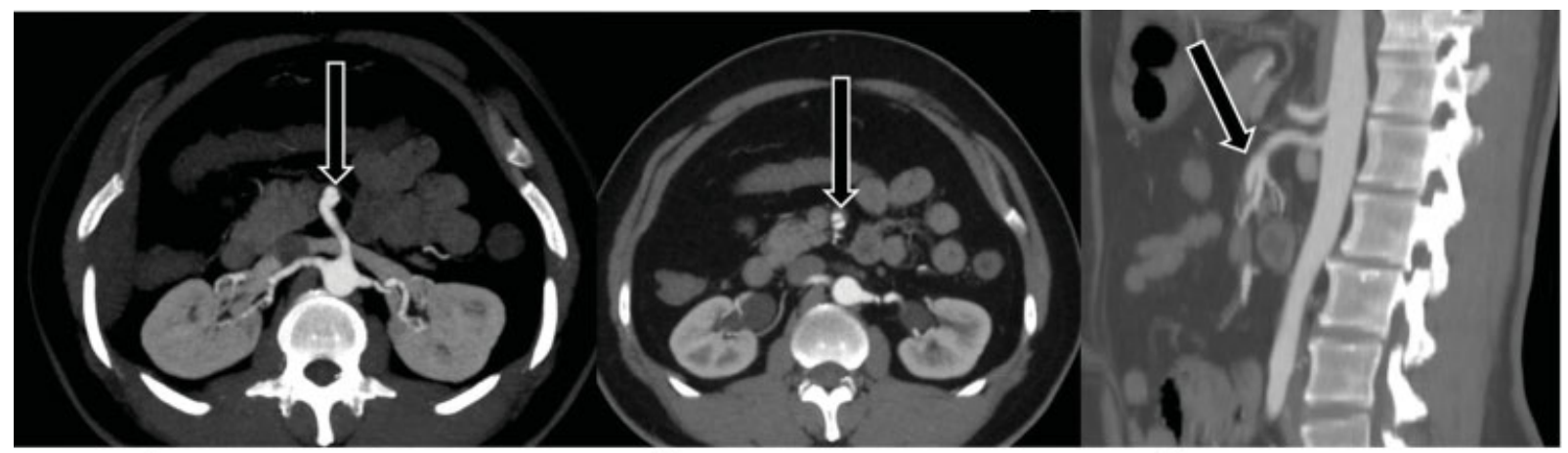

A

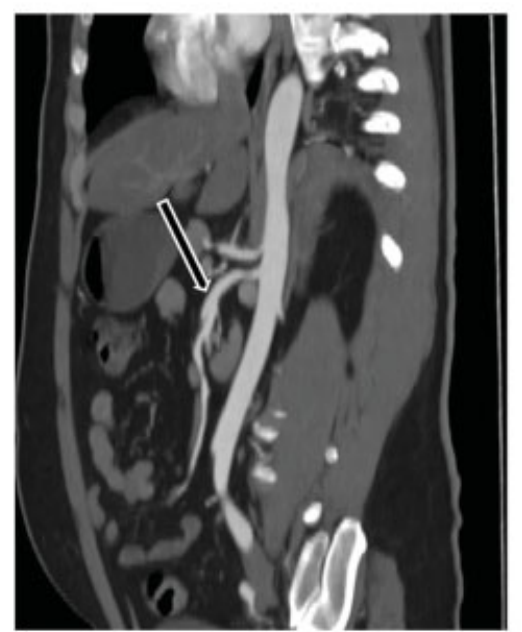

D
B

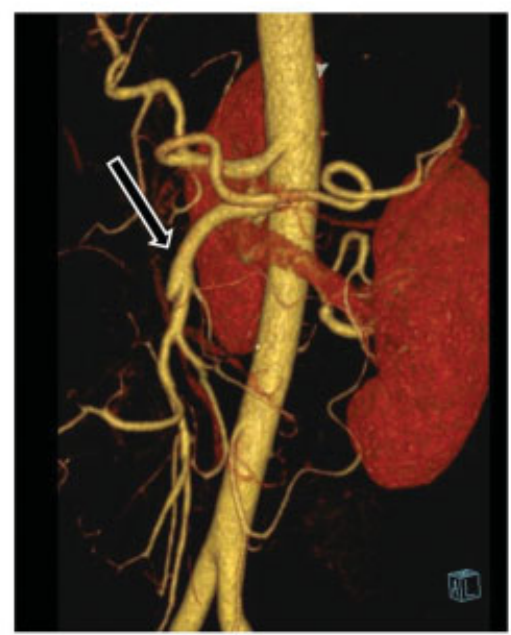

E
$\mathrm{C}$

Fig. 1 A 45-year-old male with complaints of abdominal pain and vomiting for 1 day. (A, B) Axial computed tomography (CT) images in the arterial phase showing a dissection with aneurysm (arrows) involving the proximal portion of the superior mesenteric artery, measuring $\sim 5$ mm at its maximum diameter. (C) Sagittal reformation image shows the dissecting aneurysm (arrow) in proximal superior mesenteric artery (SMA), $3.5 \mathrm{~cm}$ distal to its origin and extending for a craniocaudal length of $1 \mathrm{~cm}$. (D, E) Follow-up CT (sagittal and volume-rendering technique images) performed 4 years later (during which time the patient was on antiplatelets) shows no significant interval change in the size and extent of SMA dissection and aneurysm (arrows).

anything from incidental findings up to life-threatening hemorrhages. The most common presentation is acute abdominal pain and distension, intra-abdominal hemorrhaging with a corresponding fall in hematocrit levels, hypotension, and features of bowel ischemia. Flank pain with hematuria and deranged renal function can also be observed with renal artery involvement. ${ }^{2}$ Less commonly, cerebral artery involvement has been noted in young adults who often present with intense headaches, dizziness, visual disturbances, and sudden cerebrovascular accidents. ${ }^{2,6}$ Neonates can present with hypoxia in cases of coronary artery involvement. $2,6,7$

SAM usually involves the medium-sized splanchnic vessels and more commonly involves the celiac arteries, the SMA, and the renal arteries. ${ }^{2,8}$ The principal hallmark imaging findings are dissection with intramural hematoma or thrombosis and fusiform or saccular aneurysms. ${ }^{6,8}$ Other commonly observed imaging findings are circumferential or eccentric wall thickening with subsequent vessel occlusion or stenosis. Alternate segments of narrowing and dilatation have also been noted in a few cases, although this type of involvement is difficult to differentiate from FMD. SAM aneurysms can appear randomly without any predilection for bifurcation sites, unlike mycotic aneurysms. ${ }^{2,9}$

Contrast-enhanced CT can provide a reliable diagnosis of $\mathrm{SAM},{ }^{9}$ while catheter angiography (digital subtraction angiography) reveals the luminal pathology more clearly. However, CT has certain advantages over catheter angiography as it can detect vessel wall thickening and hematomas. CT can also detect other intra-abdominal pathologies such as mesenteric or retroperitoneal hematoma, bowel wall thickening, and pneumatosis and end-organ infarcts (e.g., renal and splenic infarcts). ${ }^{2}$ As it is noninvasive, CT is the preferred diagnostic modality for suspected SAM and also plays an important role in follow-up and treatment. Magnetic resonance angiography, despite being noninvasive and nonionizing, is only of limited value due to its suboptimal visualization of the smaller distal branches. ${ }^{8}$ Histopathological examination is the gold standard for the diagnosis of SAM, ${ }^{5,9,10}$ but it is only available for cases that require surgical resection, which does not happen often.

SAM must be differentiated from other inflammatory vascular diseases such as PAN, Takayasu's arteritis, Behçet's disease, and Henoch Schönlein purpura. This is because the 


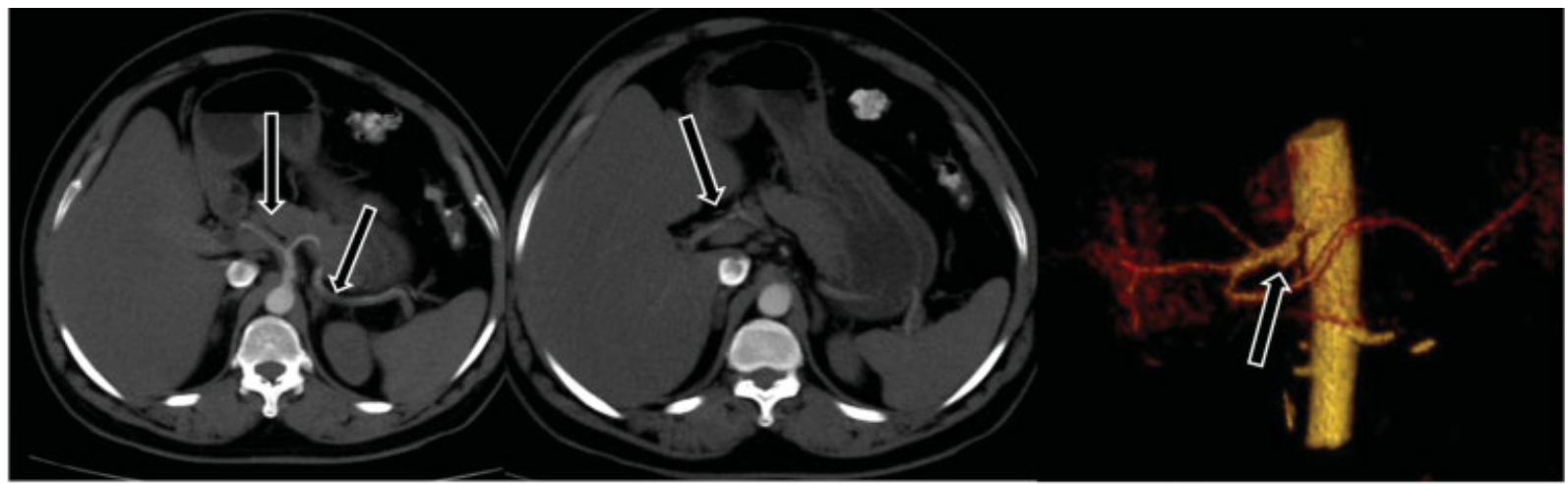

A

B

$\mathrm{C}$

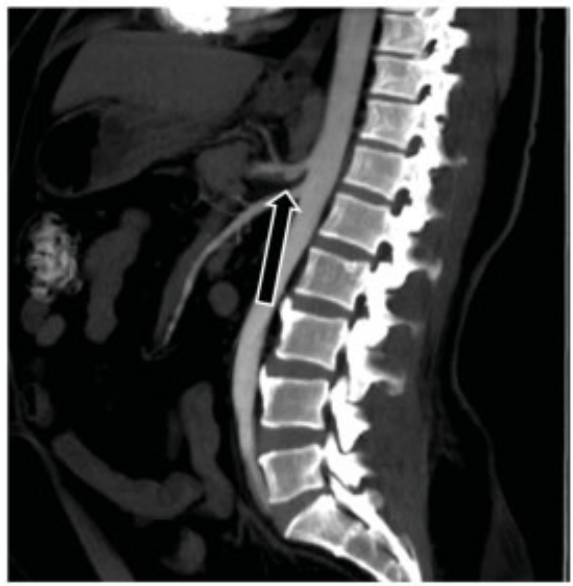

$\mathrm{D}$

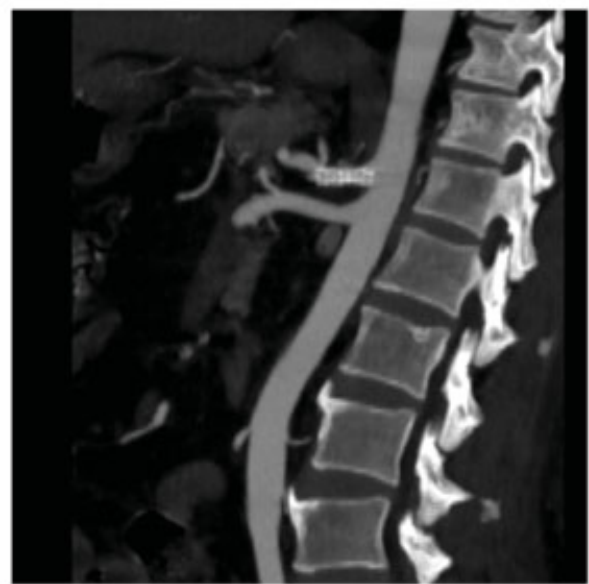

E

Fig. 2 A 50-year-old male presented with abdominal pain. (A, B) Axial contrast-enhanced computed tomography images in arterial phase showing circumferential vessel wall thickening with intramural hematoma and severe luminal narrowing involving celiac, common hepatic, proper hepatic, and splenic arteries (arrows). (C) Volume-rendering technique image from the arterial phase showing a focal dissecting aneurysm (arrow) involving the inferior wall of the celiac artery. (D, E) Sagittal maximum intensity projection images showing pre- and poststent grafting of the celiac artery dissecting aneurysm.

clinical profile of the associated features and laboratory parameters, including elevated nonspecific markers of inflammation and autoantibodies, often favors a diagnosis of vasculitis rather than SAM. ${ }^{2,9}$ Histological tests show inflammatory cells in the vessel wall in cases of vasculitis, whereas in SAM, inflammation is minimal or absent in the vessel wall. However, necrosis and vacuolar degeneration can occur in the outer layers of the arterial media, which consequently become separated from the adventitia. ${ }^{4,10}$ Since histology is rarely used due to the rarity of SAM cases requiring surgical resection, clinical profiles and laboratory parameters play a major role in the differentiation of SAM from other similar diseases.

Congenital structural vascular and connective tissue disorders such as neurofibromatosis type I, Ehlers-Danlos syndrome type IV (vascular type), and Marfan syndrome should also be considered in differential diagnoses. Imaging findings of these pathologies include arterial stenosis, dissection of the aorta and its branches due to collagen deficiency, and cystic medial degeneration leading to aortic aneurysm. 4,9,10

Another differential diagnosis is FMD that is also a nonarteriosclerotic noninflammatory condition affecting medium-sized vessels with similar radiological and histological findings. However, it differs in terms of age of onset, gender, distribution of the affected arteries, symptoms, and treatment. FMD predominantly affects middle-aged women with a predilection for renal, extracranial carotid, and vertebral arteries, whereas SAM has no age or gender predilection and most commonly involves visceral arteries like the mesenteric and celiac arteries. ${ }^{11}$

SAM usually has an acute presentation, with abdominal pain or intra-abdominal hemorrhage and derangement of renal function in cases of renal artery involvement, whereas FMD is asymptomatic except in some cases who may present with hypertension. ${ }^{8}$ Though most of the literature suggests that FMD and SAM are two separate entities, some researchers believe that both of them are at different ends of the spectrum of the same disease. ${ }^{8}$ Another school of thought is that SAM may be a precursor ${ }^{12}$ or a variant of FMD. ${ }^{12-14}$

SAM usually has a self-limiting course, with vascular remodeling and healing occurring over the course of a few weeks or months. Symptoms are mainly caused by visceral ischemia or hemorrhage, and the patients are usually managed conservatively with antiplatelets/anticoagulants. ${ }^{8}$ Endovascular intervention by stenting or stent-grafting of the vessel and/or coiling of the aneurysm or bleeding vessel 


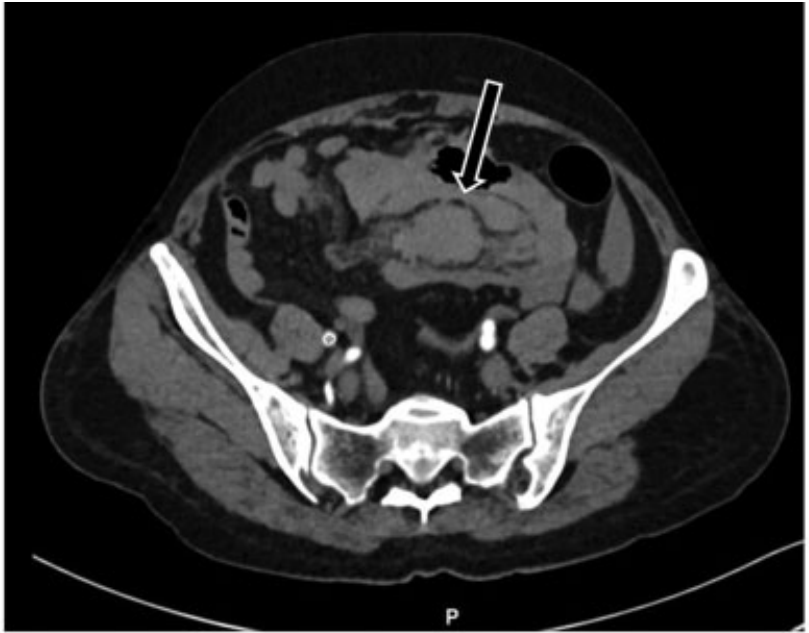

A

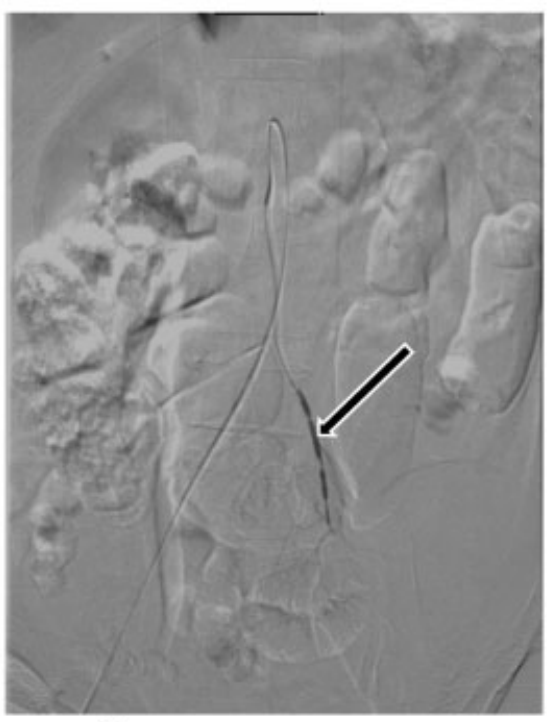

$\mathrm{C}$

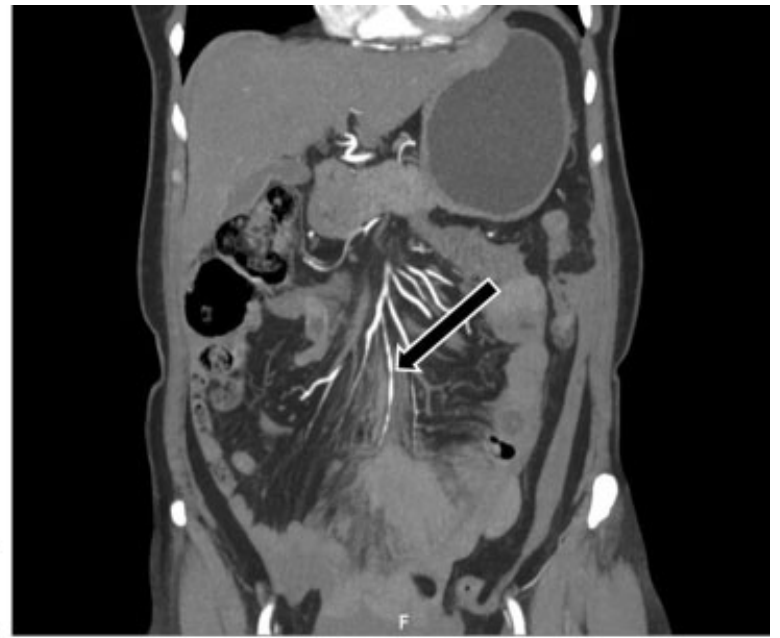

B

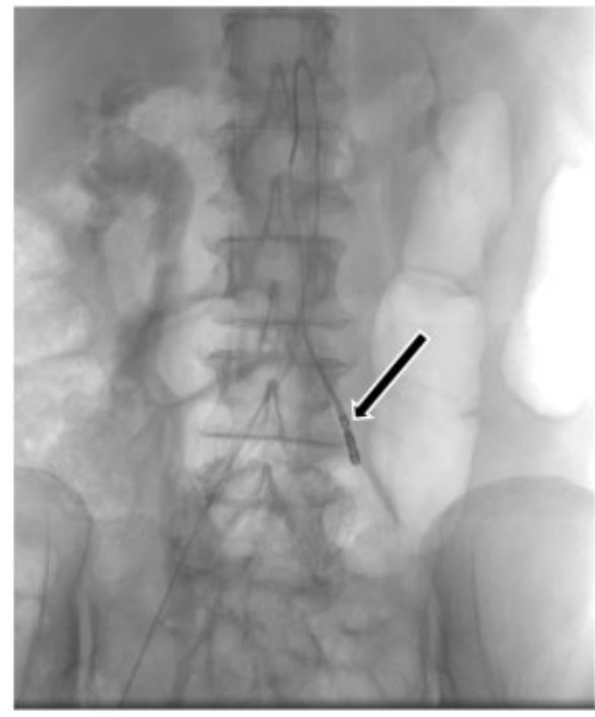

D

Fig. 3 A 62-year-old female presented with c/o abdominal pain for 2 days. (A) Contrast-enhanced computed tomography image in axial plane showing a hematoma in the small bowel mesentery (arrow) in the midline. (B) Coronal reformatted image showing a hematoma in the small bowel mesentery and beaded appearance of one of the jejunal branches of superior mesenteric artery (arrow). (C) Super selective digital subtraction angiography of the abnormal jejunal branch(arrow). (D) Fluoroscopy image showing coils within the abnormal branch (arrow).

may be needed in cases that cannot be managed medically or in cases of hemodynamic instability. ${ }^{2}$ When an aneurysm is incidental and more than $10 \mathrm{~mm}$, it can be treated by endovascular means. ${ }^{15}$ If the vessel is expendable, such as if it is a jejunal or ileal branch of SMA or is the gastroduodenal artery, then the lesion is crossed and trapped with coils. If the vessel is vital, then reconstruction with a stent (in a critically stenosed vessel) or a stent graft (in a dissecting aneurysm) is performed instead. ${ }^{2}$ All patients with acute hemorrhage require interventional treatment. Surgical resection of the end organ secondary to infarction or a surgical bypass of the stenotic vessel may also be considered in appropriate cases. ${ }^{2}$

The mortality rate in the earlier literature was reported to be up to $50 \%^{10,15,16}$ in acute presentations with intra-abdominal hemorrhage. However, recent advances in endovascular/surgical interventions have reduced the mortality rate to $\sim 25 \%,{ }^{10}$ and all the patients in our case study survived. Patients who survive the initial acute phase are likely to have no major complications at follow-up appointments, with stabilization or regression of the disease. Only a small percentage of follow-up patients shows disease progression, typically in the form of an increase in the size of the aneurysm, propagation of the dissection, or the development of new aneurysms and dissections. ${ }^{2,6,9}$

\section{Conclusion}

SAM should be considered as a potential reason for acute abdominal pain with splanchnic vascular findings of dissection or aneurysms when other causes are excluded, as well as in cases of unexplained abdominal hemorrhaging. Radiologists need to be vigilant to the possibility to detect it early, alert the clinician, and guide appropriate management. 


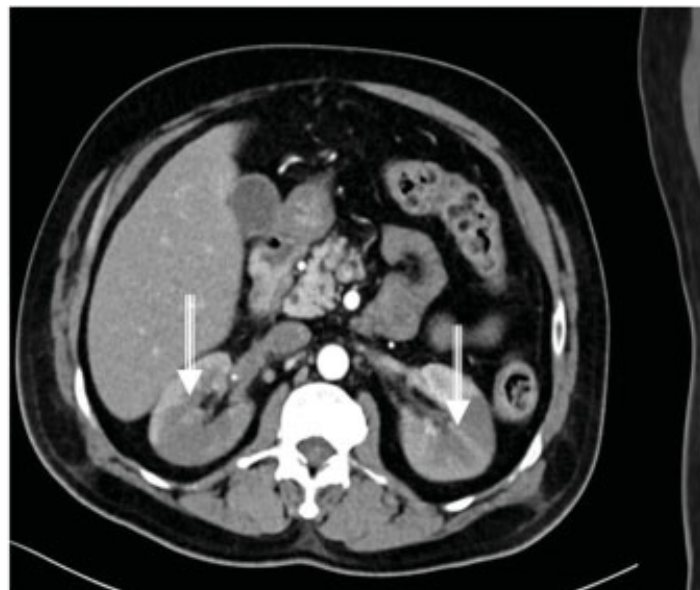

A

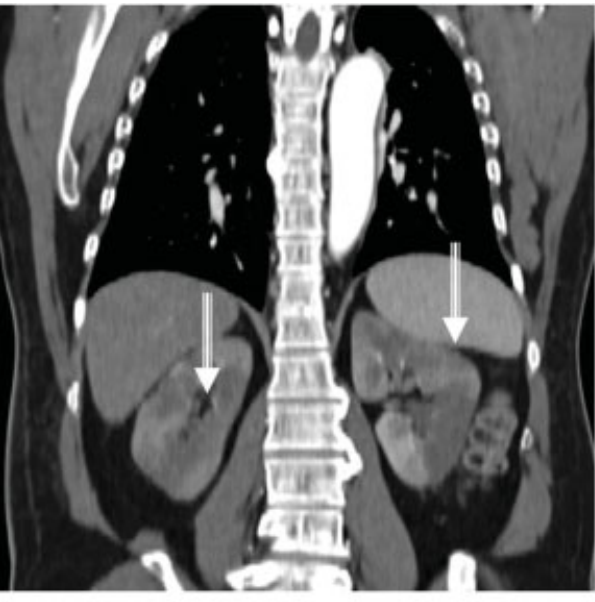

B

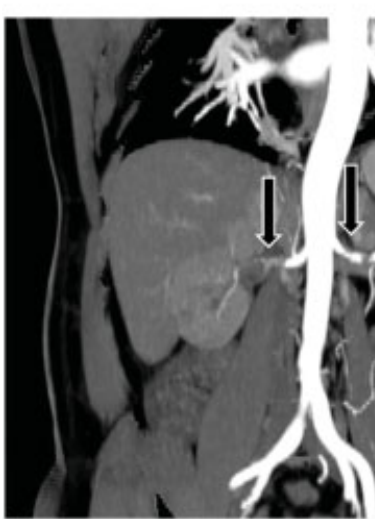

$\mathrm{C}$

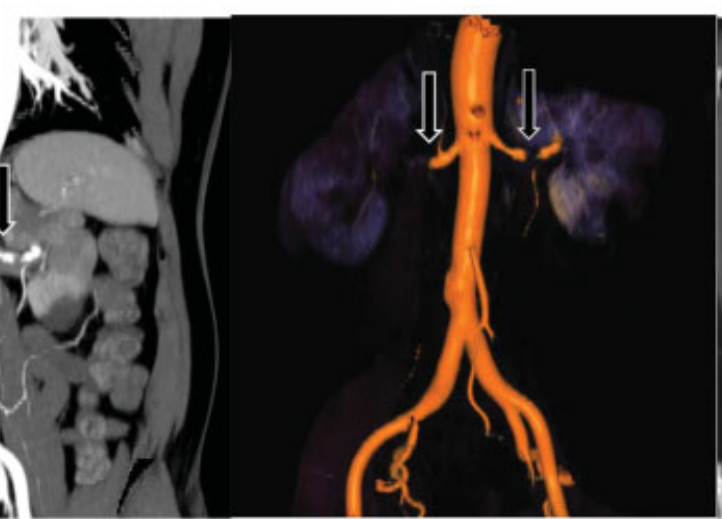

D

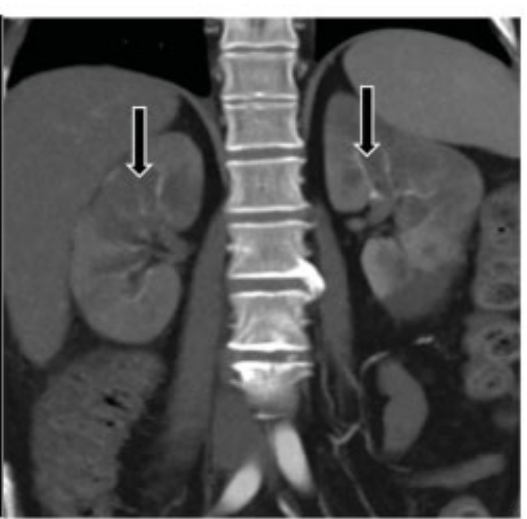

E

Fig. 4 A 50-year-old male presented with abdominal pain and worsening renal failure. (A, B) Axial and coronal contrast-enhanced computed tomography images in arterial phase showing multiple and bilateral renal infarcts (white arrows). (C, D) Coronal maximum intensity projection (MIP) and volume-rendering technique images showing dissection with intraluminal thrombus (black arrows) in the mid segment of bilateral renal arteries. (E) Coronal MIP images showing the attenuated caliber (arrows) of distal branches with normal proximal and ostial portions.

Table 1 A summary of 13 cases

\begin{tabular}{|l|l|l|l|}
\hline S. no. & Age/Sex & Contrast-enhanced CT findings & Management \\
\hline 1 & $29 / \mathrm{M}$ & Bilateral renal artery dissecting aneurysms & Stent-grafting of left renal artery \\
\hline 2 & $44 / \mathrm{M}$ & $\begin{array}{l}\text { Beaded appearance and stenosis of the hepatic artery and a } \\
\text { dissecting aneurysm of the celiac axis }\end{array}$ & $\begin{array}{l}\text { Stent-grafting of dissecting } \\
\text { aneurysm of celiac trunk }\end{array}$ \\
\hline 3 & $45 / \mathrm{M}$ & Dissection with thrombosis of a false lumen in the SMA & Conservative management \\
\hline 4 & $50 / \mathrm{M}$ & $\begin{array}{l}\text { Focal dissecting aneurysm in the celiac artery with intramural } \\
\text { hematomas in the celiac artery and the common and proper hepatic } \\
\text { artery }\end{array}$ & $\begin{array}{l}\text { Stent-grafting of celiac artery } \\
\text { dissecting aneurysm }\end{array}$ \\
\hline 5 & $75 / \mathrm{M}$ & $\begin{array}{l}\text { Aneurysmal dilatation and short segment dissection in the celiac } \\
\text { and SMAs }\end{array}$ & Conservative management \\
\hline 6 & $52 / \mathrm{M}$ & $\begin{array}{l}\text { Circumferential thickening with luminal narrowing in the celiac } \\
\text { trunk }\end{array}$ & Conservative management \\
\hline 7 & $52 / \mathrm{M}$ & $\begin{array}{l}\text { Saccular aneurysm arising from the celiac artery, with a cuff of soft } \\
\text { tissue around the celiac and hepatic arteries }\end{array}$ & Conservative management \\
\hline 8 & $53 / \mathrm{M}$ & $\begin{array}{l}\text { Aneurysm with partial thrombosis of the proximal SMA with a small } \\
\text { dissection flap }\end{array}$ & Conservative management \\
\hline 9 & $46 / \mathrm{M}$ & $\begin{array}{l}\text { Short segment wall thickening with luminal narrowing involving the } \\
\text { proximal SMA }\end{array}$ & Conservative management \\
\hline
\end{tabular}


Table 1 (Continued)

\begin{tabular}{|l|l|l|l|}
\hline S. no. & Age/Sex & Contrast-enhanced CT findings & Management \\
\hline 10 & $35 / \mathrm{M}$ & $\begin{array}{l}\text { Fusiform, nonthrombosed aneurysm in the left main renal artery } \\
\text { with adjacent retroperitoneal hematoma and mild hemoperitoneum } \\
\text { Small saccular aneurysm of the right distal main renal artery } \\
\text { Partial thrombus with focal narrowing of the left gastric artery and } \\
\text { aneurysmal dilatation of the replaced left hepatic artery }\end{array}$ & Conservative management \\
\hline 11 & $62 / \mathrm{F}$ & $\begin{array}{l}\text { Acute hematoma within the ileal mesentery with an interval } \\
\text { increase in size and mild hemoperitoneum. Beaded appearance of } \\
\text { one of the jejunal branches of the SMA }\end{array}$ & Embolization of jejunal branch \\
\hline 12 & $50 / \mathrm{M}$ & $\begin{array}{l}\text { Thrombosis with dissection of the middle segment of the bilateral } \\
\text { renal arteries. A reduced caliber of the distal branches and bilateral } \\
\text { renal infarcts }\end{array}$ & Conservative management \\
\hline 13 & $53 / \mathrm{M}$ & $\begin{array}{l}\text { Dissection with an intramural hematoma in the celiac artery } \\
\text { extending to the splenic and hepatic branches }\end{array}$ & Conservative management \\
\hline
\end{tabular}

Abbreviations: CT, computed tomography; SMA, superior mesenteric artery.

Source of Support (If present, give more details)

Nil.

\section{Presentation at a Meeting}

Nil.

\section{Conflict of Interest \\ None declared.}

\section{Acknowledgment}

Nil.

\section{References}

1 Slavin RE, Gonzalez-Vitale JC. Segmental mediolytic arteritis: a clinical pathologic study. Lab Invest 1976;35(01):23-29

2 Chao CP. Segmental arterial mediolysis. Semin Intervent Radiol 2009;26(03):224-232

3 Slavin RE, Inada K. Segmental arterial mediolysis with accompanying venous angiopathy: a clinical pathologic review, report of 3 new cases, and comments on the role of endothelin-1 in its pathogenesis. Int J Surg Pathol 2007;15(02):121-134

4 Horsley-Silva JL, Ngamruengphong S, Frey GT, Paz-Fumagalli R, Lewis MD. Segmental arterial mediolysis: a case of mistaken hemorrhagic pancreatitis and review of the literature. JOP 2014;15(01):72-77

5 Baker-LePain JC, Stone DH, Mattis AN, Nakamura MC, Fye KH. Clinical diagnosis of segmental arterial mediolysis: differentiation from vasculitis and other mimics. Arthritis Care Res (Hoboken) 2010;62(11):1655-1660
6 Kalva SP, Somarouthu B, Jaff MR, Wicky S. Segmental arterial mediolysis: clinical and imaging features at presentation and during follow-up. J Vasc Interv Radiol 2011;22(10):1380-1387

7 Gruenwald P. Necrosis in the coronary arteries of newborn infants. Am Heart J 1949;38(06):889-897, illust

8 Naidu SG, Menias CO, Oklu R, et al. Segmental arterial mediolysis: abdominal imaging of and disease course in 111 patients. AJR Am J Roentgenol 2018;210(04):899-905

9 Michael M, Widmer U, Wildermuth S, Barghorn A, Duewell S, Pfammatter T. Segmental arterial mediolysis: CTA findings at presentation and follow-up. AJR Am J Roentgenol 2006;187(06): 1463-1469

10 Garg N, Keller FS. Segmental arterial mediolysis, a vasculitis mimic: case report and review of literature. IJDI 2014;1:101-105

11 Ko M, Kamimura K, Sakamaki A, et al. Rare mesenteric arterial diseases: fibromuscular dysplasia and segmental arterial mediolysis and literature review. Intern Med 2019;58(23):3393-3400

12 Slavin RE, Saeki K, Bhagavan B, Maas AE. Segmental arterial mediolysis: a precursor to fibromuscular dysplasia? Mod Pathol 1995;8(03):287-294

13 Lie JT. Segmental mediolytic arteritis. Not an arteritis but a variant of arterial fibromuscular dysplasia. Arch Pathol Lab Med 1992; 116(03):238-241

14 Armas OA, Donovan DC. Segmental mediolytic arteritis involving hepatic arteries. Arch Pathol Lab Med 1992;116(05):531-534

15 Soulen MC, Cohen DL, Itkin M, Townsend RR, Roberts DA. Segmental arterial mediolysis: angioplasty of bilateral renal artery stenoses with 2-year imaging follow-up. J Vasc Interv Radiol 2004;15(07):763-767

16 Sakano T, Morita K, Imaki M, Ueno H. Segmental arterial mediolysis studied by repeated angiography. Br J Radiol 1997;70(834): 656-658 